\title{
Frequency-dependent spin susceptibility in the two-dimensional Hubbard model
}

\author{
C.E. Creffield, P.E. Kornilovitch, E.G. Klepfish, E.R. Pike and Sarben Sarkar \\ Department of Physics, King's College London, Strand, London WC2R 2LS, UK
}

(February 1, 2008)

\begin{abstract}
A Quantum Monte Carlo calculation of dynamical spin susceptibility in the half-filled 2D Hubbard model is presented for temperature $T=0.2 t$ and an intermediate on-site repulsion $U=4 t$. Using the singular value decomposition technique we succeed in analytically continuing the Matsubara Green's function into the real frequency domain and in deriving the spectral representation for the longitudinal and transverse spin susceptibility. The simulation results, while contradicting the random-phase approximation prediction of antiferromagnetic long-range order at this temperature, are in agreement with an extension of a self-consistent renormalization approach of Moriya. The static susceptibility calculated using this technique is qualitatively consistent with the $\omega \rightarrow 0$ simulation results.
\end{abstract}

PACS numbers: 74.20.-z, 74.20.Mn, 74.25.Dw

The dynamical magnetic susceptibility in strongly correlated electron systems (SCES) has been at the centre of attempts to explain the pairing mechanism in high temperature superconductivity (HTSC) [1], [2] as well as to understand the normal-state properties of the HTSC compounds [3], [4]. The frequency dependence of the magnetic susceptibility can be measured in neutron scattering experiments, thus allowing the assessment of the relevance of various low-energy models of SCES to HTSC, and the validity of their approximate solutions in various temperature and doping regimes. Quantum Monte Carlo (QMC) simulations, among other numerical techniques, provide an alternative test of these models and of the applicability of the analytical approximations. These simulations supply direct information only about the imaginary-time dependence of the correlation functions. Therefore, most of the information derived from them has been concerned with the static susceptibility 57. Analytic continuation of QMC data into the realtime domain has been performed recently to obtain the single-particle spectral weight function [6], [7] and twoparticle [8] Green's functions. However, a comprehensive dynamical description of the SCES, particularly of their collective excitations, based on QMC simulations, remains a largely unexplored area. In this letter we report results of analytic continuation by means of singular value decomposition (SVD) to evaluate the dynamical spin susceptibility. These results allow us to examine spectral characteristics predicted in the spin-density wave (SDW) treatment 11 for a 2D Hubbard model at finite temperature.

Interpretations of QMC results for magnetic susceptibility have been mostly related to random-phase approximation (RPA) calculations which lead to an SDW ground state [1]. Comparisons have been made between finitelattice simulations and RPA results for an infinite system [5], [9], thus neglecting the finite-size effects. These latter calculations overestimate the value of the Néel temper- ature [10] which would be only enhanced in a calculation done on a finite lattice [11]. Even without this enhancement, the fit of the QMC data to the RPA prediction requires an artificial correction to the bare Coulomb repulsion in the RPA expression for the susceptibility [5], 11], 12. We explored, therefore, an application of an extended version of the self-consistent renormalization (SCR) theory which is known to provide a more accurate description of weak antiferromagnetism [10], and found that for a finite system this theory agrees qualitatively with the QMC results with no parameter-adjustment.

We apply a finite-temperature QMC algorithm simulating the partition function of the Hubbard model as a path integral of a euclidean field theory. The temperature is represented by an additional compact dimension whose extent is equal to the inverse temperature $\beta$. Matsubara Green's functions $G_{A B}(\tau)$ for operators $A$ and $B$, satisfying for imaginary-time separation $\tau G(\tau+\beta)= \pm G(\tau)$ are evaluated as ensemble averages of the type:

$$
G_{A B}(\tau)=\frac{\int[D \Phi] A(\tau) B(0) \exp (S[\Phi])}{\int[D \Phi] \exp (S[\Phi])} ; \quad(0<\tau \leq \beta)
$$

where $\Phi$ are the generalised coordinates of the action $S$. Analytic continuation of these functions into real time amounts to a solution of the following ill-posed inverse problem:

$$
G(\tau)=\int_{-\infty}^{\infty} d \omega \frac{e^{-\omega \tau}}{1 \pm e^{-\omega \beta}} \chi^{\prime \prime}(\omega) ; \quad(0<\tau \leq \beta) .
$$

In what follows $\chi^{\prime \prime}(\omega)$ is the imaginary part of the commutator retarded Green's function, which determines the sign in the denominator $(-)$ and the periodicity of the boundary condition. The solution for $\chi^{\prime \prime}(\omega)$ was found using the singular value decomposition technique described in Ref. [7]. 
We calculate spin-correlation functions derived from QMC simulation data for Matsubara Green's functions of the type:

$$
\begin{aligned}
& G_{S^{z} S^{z}}(\vec{k}, \tau)=\left\langle\left(n_{\uparrow}(\vec{k}, \tau)-n_{\downarrow}(\vec{k}, \tau)\right)\left(n_{\uparrow}(-\vec{k}, 0)-n_{\downarrow}(-\vec{k}, 0)\right)\right\rangle \\
& G_{S^{+} S^{-}}(\vec{k}, \tau)=\left\langle c_{\uparrow}^{\dagger}(\vec{k}, \tau) c_{\downarrow}(\vec{k}, \tau) c_{\downarrow}^{\dagger}(-\vec{k}, 0) c_{\uparrow}(-\vec{k}, 0)\right\rangle
\end{aligned}
$$

where $c_{\sigma}^{\dagger}\left(c_{\sigma}\right)$ is an electron creation (annihilation) operator with spin projection $\sigma, n_{\sigma}=c_{\sigma}^{\dagger} c_{\sigma}$ and $\vec{k}$ is the lattice momentum. The simulations were performed for a single-band $2 D$ Hubbard model with on-site Coulomb repulsion $U=4 t$ ( $t$ being the nearest-neighbour hopping parameter) at simulation temperature $1 / \beta=0.2 t$ and lattice-sizes $8^{2}$ and $10^{2}$. The models were simulated at half-filling. We solve Eq.(2) with its left-hand-side given by $G_{S^{z} S^{z}}(\vec{k}, \tau)$ and $G_{S^{+} S^{-}}(\vec{k}, \tau)$, its solution $\chi^{\prime \prime}(\vec{k}, \omega)$ being the imaginary part of the dynamical longitudinal $\left(\chi^{z z}(\vec{k}, \omega)\right)$ and transverse $\left(\chi^{+-}(\vec{k}, \omega)\right)$ susceptibilities respectively.

In Fig. 1 we present the results for $\chi^{\prime \prime}(\vec{k}, \omega)$ for various values of the lattice momentum $\vec{k}$. The data is based on 4200 measurements on a $8^{2}$ lattice and 4100 measurements on a $10^{2}$ lattice. The ensemble averages and the statistical errors were estimated using bootstrap analysis. There are no qualitative differences due to finite-size effects between the two sets of results, which suggests the possibility of extrapolating the essential behaviour to the thermodynamical limit at this temperature. The analytic continuation has been based on reconstruction with seven singular functions of the kernel in Eq.(2). This number corresponds to seven singular values whose ratio to the largest one is not smaller than the average error $\left(\mathcal{O}\left(10^{-2}\right)\right)$. The Matsubara Green's functions were evaluated accordingly at seven values of $\tau$, spaced correspondinly to the zeros of the seventh singular vector.

A sum-rule used in Ref. [13] for the $t-J$ model can be applied in our case to examine the extent of doubleoccupancy in the given temperature and coupling regime. With our normalization (see Eq.(2)) this sum-rule reads:

$$
\frac{1}{N} \int_{0}^{\infty} d \omega \sum_{\vec{k}} \operatorname{coth} \frac{\beta \omega}{2} \chi^{\prime \prime z z}(\vec{k}, \omega)=\left\langle\left(S_{i}^{z}\right)^{2}\right\rangle
$$

where $N$ is the number of the lattice sites. Our results for the average on-site spin projection give $\left\langle\left(S_{i}^{z}\right)^{2}\right\rangle \approx 0.74$ which suggests that $13 \%$ of the sites are doubly occupied. This violates significantly the constraint assumed in the t-J model and undermines the validity of extrapolating results derived for the t-J model to the Hubbard model in this regime of coupling and temperature [14].

To compare our results with those derived from the SDW-RPA treatment we examine our results with respect to the evidence for long-range antiferromagnetic ordering. The SDW-RPA predicts, for the size of the systems simulated, a Néel temperature $T_{\mathrm{Neel}}^{\mathrm{RPA}} \approx 0.7 t$, which is above our simulation temperature. The same treatment predicts also an SDW gap $\Delta=1.38 t$ at our simulation temperature. This gap would result in the vanishing of the time-ordered Green's function at the nesting vector $\vec{Q}=(\pi, \pi): \chi^{\prime \prime z z T}(\vec{Q}, \omega)$ for $\omega<2 \Delta[1]$.

We find that $\chi^{\prime \prime}(\vec{k}, \omega)$ for small values of $\omega$ has a clear peak at $\vec{k}=\vec{Q}$ which leads also to a sharply enhanced real part of static susceptibility $\chi^{\prime}(\vec{Q}, \omega=0)$ calculated using the Kramers-Kronig relations (Fig.2). However our results contradict two essential SDW predictions: the spontaneous breaking of rotational symmetry and the existence of a gap in the spectral function for the magnetic correlations. The approximate preservation of the relation $\chi^{z z}(\vec{k}, \omega)=2 \chi^{+-}(\vec{k}, \omega)$ (Fig.1) indicates that the rotational symmetry is not violated, while the results for $\chi^{\prime \prime z z T}(\vec{Q}, \omega)$ (Fig.3) show no evidence for the SDW gap. This latter discrepancy with the SDW prediction cannot be explained by finite-size effects, since the sign of the gap is invariant under the change of the staggered magnetization. Therefore if antiferromagnetic order existed in the thermodynamical limit we would expect a minimum (if not vanishing) of $\chi^{\prime \prime z z T}(\vec{Q}, \omega)$ for small values of $\omega$. This is clearly absent in our results.

We observe therefore that our results cannot be adequately explained within the SDW-RPA scheme. The standard RPA result yields for $T>T_{\text {Neel }}$ :

$$
\chi_{\mathrm{RPA}}^{+-}(\vec{k}, \omega)=\frac{\chi_{0}^{+-}(\vec{k}, \omega)}{1-U \chi_{0}^{+-}(\vec{k}, \omega)} .
$$

We note that the non-interacting susceptibility $\chi_{0}(\vec{Q}, 0)$ calculated for a two-dimensional finite system at halffilling exhibits a $1 / T$ divergence as $T \rightarrow 0$. Therefore, there will always be a pole of the susceptibility at some nonzero temperature $T_{\text {Neel }}^{\mathrm{RPA}}$, indicated by $U \chi_{0}(\vec{Q}, 0)=1$, with a subsequent divergence of the total free energy of a finite system. This unphysical result is one of the consequences of an inherent shortcoming of the SDWRPA treatment, namely, its failure to represent in a selfconsistent manner the contribution of the spin fluctuations to the free energy. The free energy can be expressed as a functional in the dynamical susceptibility 110 (see Eqs.(8),(9)). On substituting expression (5) into this functional and subsequently differentiating it twice with respect to the staggered magnetization one fails to recover the static limit of (5). This inconsistency has been pointed out 10] as the reason for predicting too high values for the Néel temperature of weak-antiferromagnetic metals. These arguments suggest the need to explore an analytic approach which would more adequately describe the thermodynamics of the system, if we are to try to relate our QMC results to any analytical concepts.

Such an approach has been developed as the theory of SCR by Moriya et al. [10]. A renormalization function $\lambda$ is introduced which relates the exact sum of two-particle irreducible diagrams $\tilde{\chi}_{U M}^{+-}(\vec{k}, \omega)$ to the free susceptibility 
$\chi_{0 M}^{+-}(\vec{k}, \omega)$ in a given background staggered magnetization $M$ :

$$
\tilde{\chi}_{U M}^{+-}(\vec{k}, \omega)=\frac{\chi_{0 M}^{+-}(\vec{k}, \omega)}{1+\lambda_{U M}(\vec{k}, \omega)} .
$$

This renormalization function enters the calculation of the full susceptibility:

$$
\begin{aligned}
& \chi_{U M}^{+-}(\vec{k}, \omega)=\frac{\chi_{0 M}^{+-}(\vec{k}, \omega) \Lambda(\vec{k}+\vec{Q})+U\left[\chi_{0 M}^{+-}(\vec{k}, \vec{k}+\vec{Q}, \omega)\right]^{2}}{\Lambda(\vec{k}) \Lambda(\vec{k}+\vec{Q})-\left[U \chi_{0 M}^{+-}(\vec{k}, \vec{k}+\vec{Q}, \omega)\right]^{2}} \\
& \Lambda(\vec{k}) \equiv 1+\lambda_{U M}(\vec{k}, \omega)-U \chi_{0 M}^{+-}(\vec{k}, \omega) .
\end{aligned}
$$

Here the free umklapp susceptibility at staggered magnetization $M$ is denoted by $\chi_{0 M}^{+-}(\vec{k}, \vec{k}+\vec{Q}, \omega)$. The free energy of the system with $N_{\mathrm{el}}$ electrons is given by:

$$
F(U, M)=F_{0}(M)+\frac{U}{N}\left(\frac{N_{\mathrm{el}}^{2}}{4}-M^{2}\right)+\triangle F(U, M)
$$

where the first two terms represent mean-field free energy and the third one is the correction due to the collective excitations which can be expressed via $\chi_{U M}^{+-}(\vec{k}, \omega)$ as:

$$
\triangle F(U, M)=-T \int_{0}^{U} d U^{\prime} \sum_{\vec{k} \omega_{\nu}}\left[\chi_{U^{\prime} M}^{+-}\left(\vec{k}, i \omega_{\nu}\right)-\chi_{0 M}^{+-}\left(\vec{k}, i \omega_{\nu}\right)\right] .
$$

where $\omega_{\nu}=2 \pi T \nu$. The sum in (9) is dominated by $\chi_{U M}^{+-}(\vec{Q}, 0)$, which justifies the long-wave static approximation $\lambda_{U M}(\vec{k}, \omega)=\lambda_{U M}(\vec{Q}, 0)$. In this approximation, Eqs.(7)-(9) together with the thermodynamical relation between the static susceptibility and the free energy:

$$
\frac{1}{\chi_{U M}^{+-}(\vec{Q}, 0)}=\left.\frac{1}{2} \frac{\partial^{2} F(U, M)}{\partial M^{2}}\right|_{M=0}
$$

constitute a set of equations for the susceptibility, automatically maintaining the self-consistency requirement. Eq.(10) can be solved by expanding $\lambda_{U M}(\vec{Q}, 0)$ in powers of $M$. In the next to leading order expansion: $\lambda_{U M}(\vec{Q}, 0) \approx \lambda_{0}(U)+(1 / 2) \lambda_{2}(U) M^{2}$ we obtain:

$$
\begin{aligned}
& \lambda_{0}(U)=\left.\chi_{0 M}^{+-}(\vec{Q}, 0) \frac{\partial^{2} \Delta F}{\partial M^{2}}\right|_{M=0} \\
& \lambda_{2}(U)=\left.\frac{\partial^{2} \chi_{0 M}^{+-}(\vec{Q}, 0)}{\partial M^{2}} \frac{\partial^{2} \triangle F}{\partial M^{2}}\right|_{M=0} .
\end{aligned}
$$

Since even within this approximation the thermodynamics of the spin fluctuations is accounted for selfconsistently, the qualitative description of the susceptibility is likely to be more accurate than the one provided by SDW-RPA. Apparently due to the finite-size $1 / T$ divergence of $\chi_{0}$, Moriya's approximation of $\lambda$ independent of $U$ and $M$, used in the infinite-system calculation 10 is too crude for this calculation. By substituting the solution of Eq.(11) for $\lambda_{U M}(\vec{Q}, 0)$ into (可) at $\omega=0$ we obtain the value of $\tilde{\chi}_{U 0}^{+-}(\vec{k}, 0)$. The static susceptibility (at $T>T_{\text {Neel }}$ ) is obtained by replacing $\chi_{0}^{+-}(\vec{k}, 0)$ in Eq.(5) by $\tilde{\chi}_{U 0}^{+-}(\vec{k}, 0)$ (Fig.2). The calculation of the direct and umklapp free susceptibility entering Eq.(7) was done for lattice sizes $8^{2}$ and $10^{2}$. Thus the finite-size effects, inevitably present in the QMC simulations, have been accounted for in the analytical calculation. We note the qualitative agreement between the calculated values and the simulation results at the nesting vector and the rest of the lattice momenta. The agreement is particularly remarkable since there is no parameter adjustment in this analytical calculation. Due to the discrete spectrum of single-particle excitations on a finite lattice, $\chi_{0}^{\prime \prime}(\vec{k}, \omega)$ is a sequence of $\delta$-functions and becomes smooth only for an infinite system. An imaginary regularizer (corresponding to a finite life-time for a quasiparticle) has thus to be introduced to allow the calculation of dynamical susceptibility using Eq.(7). We found the results to be sensitive to the value of this regularizer, therefore, a meaningful comparison with the QMC data can be done only for static susceptibility.

Making a standard approximation $\chi^{\prime}(\vec{q}+\vec{Q}, 0)=$ $\chi^{\prime}(\vec{Q}, 0) /\left(1+\xi^{2} \vec{q}^{2}\right)$ for small deviations $\vec{q}$ from the nesting vector, we obtain the correlation length $\xi=3.2 a$ and $\xi=3.6 a$ ( $a$ being the lattice spacing) for the $8^{2}$ and $10^{2}$ lattices respectively. The SCR estimates are $\xi=3.9 a$ and $\xi=4.4 a$. We emphasize, however, that since the correlation length calculated is of the order of the lattice linear dimension this estimate can vary as lattice size increases.

To summarise, the SVD technique is applicable to the derivation of the dynamical properties of collective excitations in SCES. The dynamical susceptibility obtained from QMC simulations contradicts the qualitative predictions of SDW-RPA, in particular there is no evidence for SDW gap formation in the longitudinal time-ordered correlation function. Therefore it is unlikely that the gaps observed for this lattice size and temperature in previous QMC simulations of the single-particle density of states [6] can be explained within SDW-RPA as was indeed pointed out in Ref.[7]. Our results support the evidence for the insufficiency of the SDW-RPA picture to describe the spectrum of excitations of the Hubbard model [15], 16]. Their qualitative agreement in the static limit with the SCR calculation points to the importance of including the paramagnon interaction in the description of the magnetic properties of the Hubbard model. This approach has previously been used to obtain on a more phenomenological basis, the dynamics of collective excitations in HTSC compounds in the normal phase as well as to calculate the pairing potential [3]. Our work outlines a way to examine these properties directly from the lattice-field model by means of numerical simulations as well as by a power series expansion in the magnetisation within the SCR theory.

We are grateful to J.H. Jefferson, G. Aeppli, A. 
Bratkovsky and V. Kabanov for stimulating discussions. This research was funded by an SERC grant GR/J18675 and our general development of SVD techniques by the US Army Research Office, agreement no. DAAL03-92G-0142. Two of the authors (CEC and PEK) were supported by EPSRC and ORS - University of London respectively.

\section{References}

1. J.R. Schrieffer, X.W. Wen and S.C. Zhang, Phys. Rev.B39, 11663 (1989);

2. A. Kampf and J.R. Schrieffer, Phys. Rev. B41, 6399 (1990); ibid. B42, 7967 (1990);

3. T. Moriya, Y. Takahashi and K. Ueda, Jour. Phys. Soc. Japan 59, 2905 (1990);

4. P. Monthoux and D. Pines, Phys. Rev. B49, 4261 (1994);

5. N. Bulut, D.J. Scalapino and S.R. White, Phys. Rev.B47, 2742 (1993);

6. S.R. White, Phys. Rev. B44, 4670 (1991); M. Vekić and S.R. White, Phys. Rev. B47, 1160 (1993);

7. C.E. Creffield, E.G. Klepfish, E.R. Pike and Sarben Sarkar, Phys. Rev. Lett. 75, 517 (1995);

8. M. Vekić et al., Phys. Rev. Lett.74, 2367 (1995); H. Endres et al. , unpublished;

9. A. Moreo et al., Phys. Rev. B41, 2313 (1990);

10. Tôru Moriya, Spin Fluctuations in Initerant Electron Magnetism Springer-Verlag (1985); H. Hasegawa and Tôru Moriya, Jour. Phys. Soc. Japan 36, 1542 (1974);

11. L. Chen et al., Phys. Rev. Lett. 66, 369 (1991); Alain F. Veilleux et al., unpublished;

12. N. Bulut, D.J. Scalapino and S.R. White, Physica C246, 85 (1995);

13. J. Jaklic and P. Prelovsek, Phys. Rev. Lett. 75, 1340 (1995);

14. A. Moreo, Phys. Rev. B48, 3380 (1993);

15. N. Furukawa and M. Imada, J. Phys. Soc. Japan 61, 3331 (1992);

16. E.Abraham et al., unpublished;

\section{Figure Captions:}

Fig. $1 \chi^{\prime \prime z z}(\vec{k}, \omega)$ (solid line) and $\chi^{\prime \prime+-}(\vec{k}, \omega)$ (dotted line) $v s$. $\omega$ for selected values of the lattice momentum given in the brackets in each figure; (a) - lattice $8^{2}$, (b) - lattice $10^{2}$.
Fig.2 Static spin susceptibility vs. lattice momentum; (a) - lattice $8^{2},(\mathrm{~b})$ - lattice $10^{2}$. The results of the SCR calculation are shown by $(\bullet)$.

Fig.3 Time-ordered Green's function $\chi^{\prime \prime z z T}(\vec{Q}, \omega)$. Note the absence of SDW gap. 
Fig.1

(a)

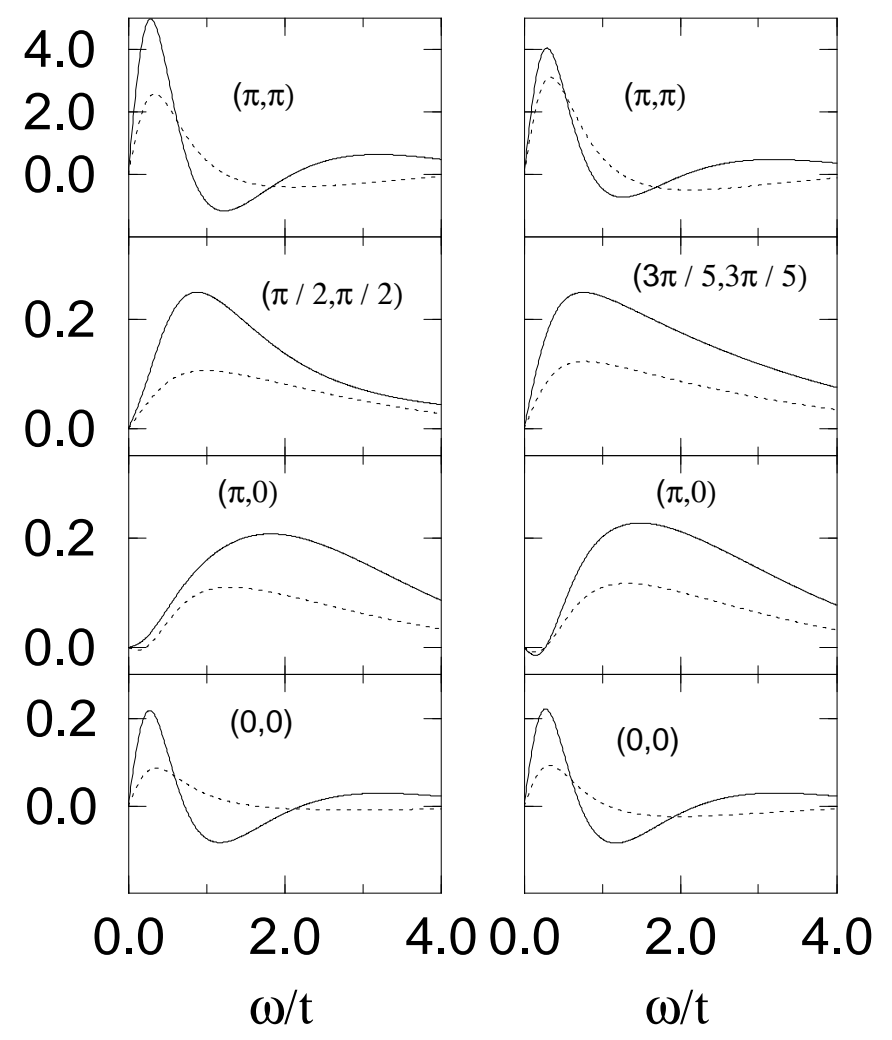

(b) 
Fig. 2
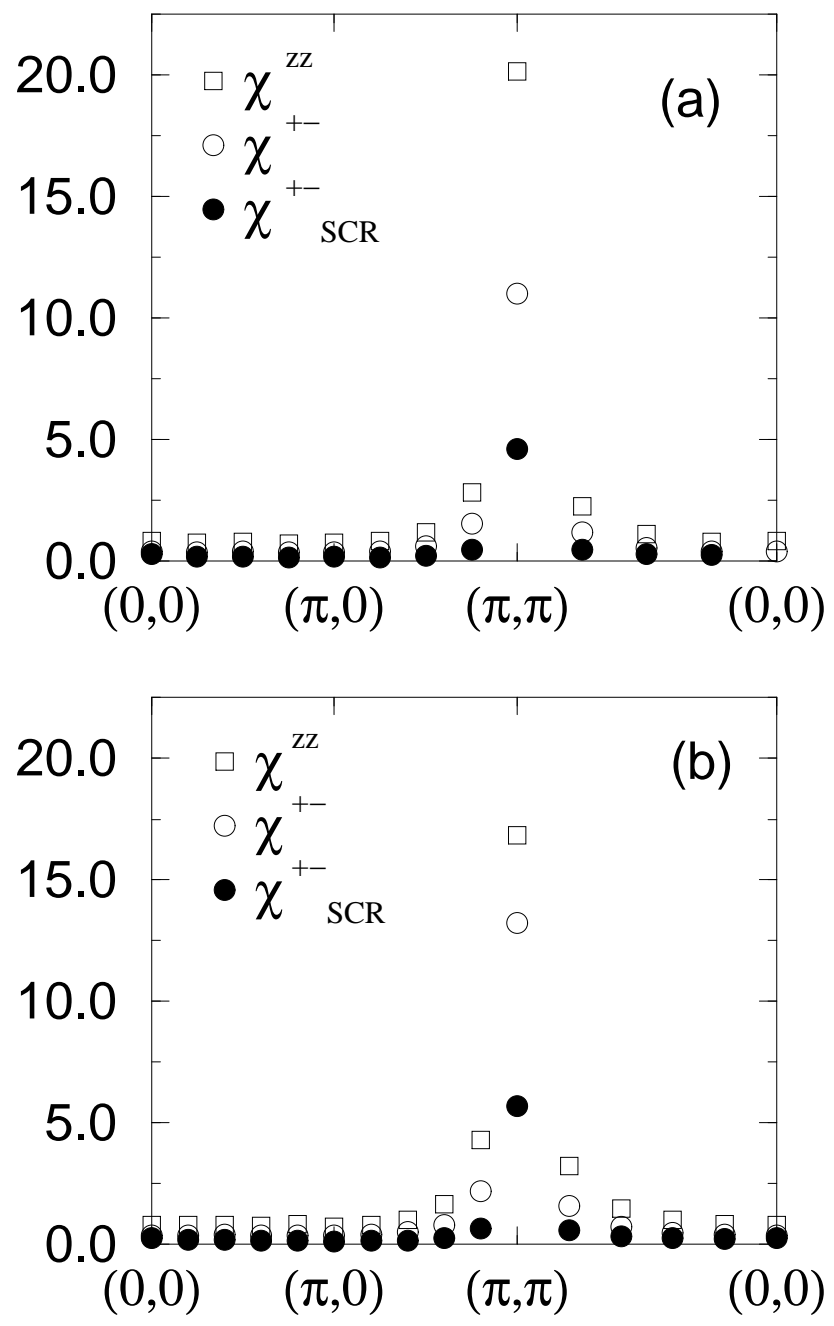
Fig. 3

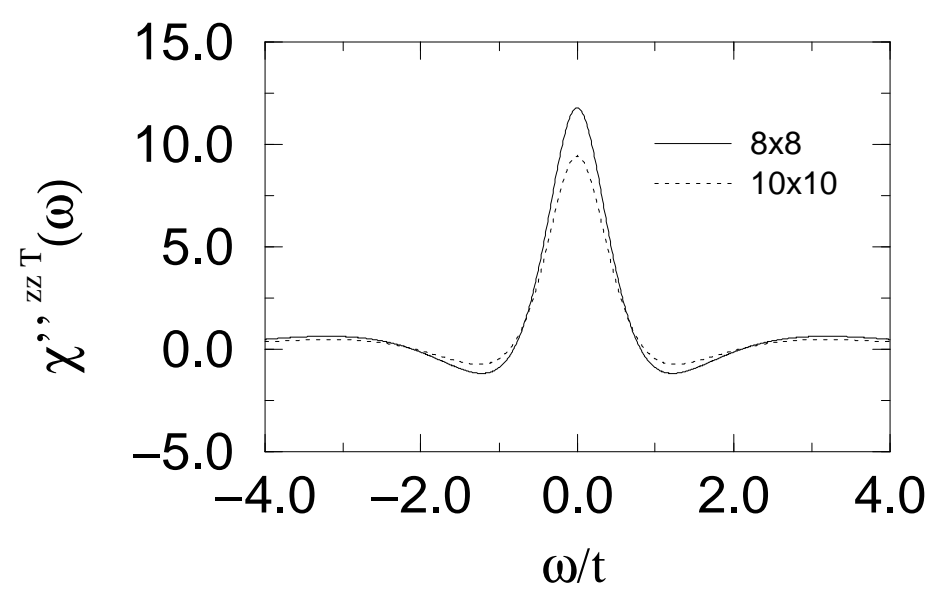

